\title{
Meta
}

Journal des traducteurs

Translators' Journal

\section{Classe et masse dans le discours syndical': formes de surface, problème de fond}

\section{Maurice Tournier}

Volume 39, numéro 4, décembre 1994

Hommage à Bernard Quemada : termes et textes

URI : https://id.erudit.org/iderudit/004500ar

DOI : https://doi.org/10.7202/004500ar

Aller au sommaire du numéro

Éditeur(s)

Les Presses de l'Université de Montréal

ISSN

0026-0452 (imprimé)

1492-1421 (numérique)

Découvrir la revue

Citer cet article

Tournier, M. (1994). Classe et masse dans le discours syndical': formes de surface, problème de fond. Meta, 39(4), 798-806.

https://doi.org/10.7202/004500ar
Résumé de l'article

Saisir les mots dans leurs rapports statistiques avec les autres mots d'un texte afin de comparer les systèmes de rapports qui caractérisent leur emploi au sein de plusieurs textes permet d'entrer dans le sens par effraction, car les mots se lisent inconsciemment dans le réseau de relations qui les articule. Un exemple de ce type d'étude est donné avec les termes masse et classe pris dans les résolutions syndicales de différentes confédérations réunies en congrès. On s'aperçoit que des spécificités à"emploi caractérisent l'un et l'autre et surtout l'un par rapport à l'autre. Absents du discours de force ouvrière et de la CFTC, masse et classe au singulier illustrent dans cet ordre une époque de la CFDT mais, dans l'ordre inverse, l'orthodoxie permanente de la CGT. Derrière la présence et l'agencement de ces formes apparaissent des options idéologiques et des intentions politiques. Est-ce le sen ? Oui, si avant de voir dans les mots des êtres de raison le lexicologue cherche dans leur emploi leurs raisons d'être. 


\title{
CLASSE ET MASSE DANS LE DISCOURS SYNDICAL : FORMES DE SURFACE, PROBLEME DE FOND
}

MAURICE TOURNIER

CNRS-INaLF, ENS de Fontenay-Saint-Cloud, Saint-Cloud, France

\begin{abstract}
Résumé
Saisir les mots dans leurs rapports statistiques avec les autres mots d' un texte afin de comparer les systèmes de rapports qui caractérisent leur emploi au sein de plusieurs textes permet d'entrer dans le sens par effraction, car les mots se lisent inconsciemment dans le réseau de relations qui les articule. Un exemple de ce type d'étude est donné avec les termes masse et classe pris dans les résolutions syndicales de différentes confédérations réunies en congrès. On s'aperçoit que des spécificités d'emploi caractérisent l'un et l'autre et surtout l'un par rapport à l'autre. Absents du discours de force ouvrière et de la CFTC, masse et classe au singulier illustrent dans cet ordre une époque de la CFDT mais, dans l'ordre inverse, l'orthodoxie permanente de la CGT. Derrière la présence et l'agencement de ces formes apparaissent des options idéologiques et des intentions politiques. Est-ce le sens? Oui, si avant de voir dans les mots des êtres de raison le lexicologue cherche dans leur emploi leurs raisons d'être.
\end{abstract}

\begin{abstract}
Apprehending words in their statistical relations with the other words of a text, comparing the system of relationships that characterize their use in several texts enables us to break into their meaning as it were, since words are to be read subconsciously in the network of relations that articulates them. An example of this kind of study is given with the terms masse and classe taken from trade union resolutions from various union council meetings. It becomes clear that specific usage characterizes both these words, and especially one in relation to the other. Masse and classe, absent from the speeches of Force Ouvrière and the CFTC, illustrate an era of the CFDT and classe and masse, in that order, are typical of the permanent orthodoxy of the CGT. Behind the presence and the order of these forms ideological options and political intentions are outlined. Is that the meaning? It is, if before seeing beings of reason in words the lexicologist sees their reason for being in their use.
\end{abstract}

Montreuil 1985, congrès national de la CGT. Au cours du débat en séance plénière, deux mots s'entrechoquent bizarrement pour une oreille exercée : classe et masse. Deux termes pourtant lourds d'une histoire commune, ou presque...

Outil du législateur romain servant à diviser le peuple d'après un critère de richesse pour en tirer des impôts, classis indiquait déjà un critère économique, le niveau du revenu. Au XVIII ${ }^{\mathrm{e}}$ siècle en France, le mot sert aux physiocrates à répartir la société en trois catégories (classes productive, propriétaire, stérile) selon les fonctions remplies ou la place occupée dans la circulation des biens'. Avec l'analyse marxiste des postes de production (mais déjà saint-simonienne ou proudhonienne), les classes ne sont plus de simples catégorisations, abstraites et multipliables; elles prennent corps dans une simplification drastique héritière des affrontements et de la conscience de faire corps qui les accompagne. «L'antagonisme entre le prolétariat et la bourgeoisie est une lutte de classe à classe» (Marx, Misère de la philosophie, 1847). Depuis, le terme s'est popularisé et marqué de cicatrices sociales. L'expression «lutte de(s) classes», constatation d'analyste chez 
Guizot en 1828 , a pu se diffuser en slogan pendant la Révolution de $1848^{2}$. C'est aussi dans cette première moitié du XIX ${ }^{\mathrm{e}}$ siècle qu'à la suite des canuts et ferrandiniers lyonnais, révoltés en 1831 et 1834 , l'usage de «classes ouvrières» passe majoritairement au singulier. Quant au figement adjectif «de classe» et au mot d'ordre «classe contre classe» ${ }^{3}$, ils apparaîtront chez Guesde autour de 1900. Malgré des emplois flous, liés à l'intrusion de «classes moyennes», classe est à cette époque une pointe aiguisée, marxisée, par les conflits sociaux de l'industrialisation.

Masse raconte un peu une histoire semblable, mais sans passer d'abord par l'économie politique et sans déboucher sur les délimitations antagoniques pointues du marxisme ${ }^{4}$. On parlait, dans la mouvance saint-simonienne, de «servir les masses» et de «l'avènement des masses», dès les années 1830 . Avec une condescendance et parfois une ironie, que classe ne suscite jamais. C'est le blanquisme (Blanqui dénonce en 1832 le pillage des «masses laborieuses» par les «oisifs») et surtout l'anarcho-syndicalisme de la fin du siècle qui retourneront en positif les expressions «la masse ouvrière», «les masses», liées à l'idée de rejet social ( $\ll$ Ces sombres masses que la bourgeoisie méprise autant qu'elle les craint», Kropotkine, 1880) mais aussi de spontanéité révolutionnaire et de grève générales. Valeur devenue glorieuse en discours socialiste: «grève de masse» calquera le «Massenstreik» des Spartakistes ${ }^{6}$. Les deux termes sont liés, on le voit, à la même histoire. Devenus l'apanage du discours communiste des années trente (Front unique de classe et Front unique de masse alternent dans L'Humanité au début de 1934), classe et masse refleuriront dans les tracts gauchistes de Mai 68; mais une diversité dans l'insistance n'y est pas sans rapport avec d'anciens clivages communistes / anarchistes...

C'est en tenant compte de ce passé social chargé qu'il faut apprécier leurs emplois - ou leurs non-emplois - dans le discours officiel tenu par les confédérations ouvrières actuelles, CGT, CFDT, FO et CFTC. Que nous disent à leur propos l'ensemble des constats fréquentiels systématiquement pratiqués sur les différentes formes qu'ils prennent dans les textes?

\section{ÉTUDE DU CHAMP : UN CLIVAGE INTERCONFÉDÉRAL}

L'ensemble des résolutions nationales votées à la majorité dans les quatre centrales ouvrières entre 1971 et 1990, soit pendant vingt années de congrès confédéraux, a été enregistré en machine par les soins de J. Lefèvre et du laboratoire de Saint-Cloud7. Sur cet énorme corpus de données (751 119 occurrences de 16724 formes minimales), structuré en deux parties de dix ans ou en 27 congrès, appliquons l'analyse dénommée au laboratoire «Calcul des spécificités»8. Chaque sous-fréquence f constatée localement y est jaugée en probabilité par rapport au corpus, à la fréquence totale $(F)$ et à la partition en deux sousensembles de quatre centrales (Voir le tableau 1).

Avec ces quatre formes, le discours dispose d'un outil de clivage certain. La CGT surtout et la CFDT (qui cumulent 6 indices positifs à très forts coefficients de spécificité) s'opposent radicalement à FO et surtout à la CFTC (qui cumulent 8 indices négatifs) 9 . Cette opposition est d'autant plus évidente que leurs rares occurrences dans le discours syndical chrétien soit appartiennent à un domaine, classes scolaires ou masses salariales, autre que celui des désignations sociales qui nous occupe ici, soit constituent des rejets parodiques du discours adverse : la CFTC stigmatise «les partisans de la lutte des classes». Â quelques exceptions près, FO évite, elle aussi, ce type de vocabulaire. D'ailleurs, ses occurrences de classes en relation avec l'école, une fois décomptées, font disparaitre la seule spécificité positive que l'ordinateur ait mise à son actif 10 .

Aussi évidente est la baisse proportionnelle des fréquences dans le temps, que caractérise le passage, pour CGT et CFDT, de spécificités positives très fortes en première période à des spécificités négatives ou des indices de banalité en seconde période. Ainsi, 
Tableau 1:

Classe(s), Masse( $(s)$ : ventilation de la fréquence $\mathrm{F}$ et probabilisation des sous-fréquences $\mathrm{f}$

\begin{tabular}{|c|c|c|c|c|c|c|c|c|c|}
\hline & $\begin{array}{l}\text { CFDT } \\
73-79 \\
43042\end{array}$ & $\begin{array}{l}\text { CFDT } \\
82-88 \\
29704\end{array}$ & $\begin{array}{l}\text { CFTC } \\
71-79 \\
42266\end{array}$ & $\begin{array}{l}\text { CFTC } \\
81-90 \\
92239\end{array}$ & $\begin{array}{l}\text { CGT } \\
72-78 \\
127961\end{array}$ & $\begin{array}{l}\mathrm{CGT} \\
82-89 \\
316352\end{array}$ & $\begin{array}{l}\text { FO } \\
71-80 \\
69613\end{array}$ & $\begin{array}{l}\text { FO } \\
84-89 \\
29942\end{array}$ & $\begin{array}{l}\mathrm{F} \\
71-90 \\
751119\end{array}$ \\
\hline classe & $\begin{array}{l}S+38 \\
116\end{array}$ & $\begin{array}{l}\text { b } \\
14\end{array}$ & $\begin{array}{l}\text { S-13 } \\
0\end{array}$ & $\begin{array}{l}\text { S-27 } \\
1\end{array}$ & $\begin{array}{l}\text { S+06 } \\
127\end{array}$ & $\begin{array}{l}\text { b } \\
218\end{array}$ & $\begin{array}{l}\text { S-06 } \\
19\end{array}$ & $\begin{array}{l}\mathrm{S}-\mathbf{0 3} \\
8\end{array}$ & 503 \\
\hline masse & $\begin{array}{l}S+17 \\
66\end{array}$ & $\begin{array}{l}\text { S-02 } \\
7\end{array}$ & $\begin{array}{l}\text { S-08 } \\
1\end{array}$ & $\begin{array}{l}\text { S-14 } \\
6\end{array}$ & $\begin{array}{l}\mathbf{S + 0 9} \\
106\end{array}$ & $\begin{array}{l}\text { b } \\
164\end{array}$ & $\begin{array}{l}\text { S-07 } \\
9\end{array}$ & $\begin{array}{l}\text { S-06 } \\
1\end{array}$ & 360 \\
\hline classes & $\begin{array}{l}\text { b } \\
9\end{array}$ & $\begin{array}{l}\text { S-02 } \\
0\end{array}$ & $\begin{array}{l}\mathbf{b} \\
2\end{array}$ & $\begin{array}{l}\text { b } \\
6\end{array}$ & $\begin{array}{l}\text { S+02 } \\
24\end{array}$ & $\begin{array}{l}\text { b } \\
32\end{array}$ & $\begin{array}{l}S+\mathbf{0 2} \\
14\end{array}$ & $\begin{array}{l}\text { b } \\
5\end{array}$ & 92 \\
\hline masses & $\begin{array}{l}\text { b } \\
5\end{array}$ & 0 & 0 & $\begin{array}{l}\text { b } \\
2\end{array}$ & $\begin{array}{l}S+11 \\
26\end{array}$ & $\begin{array}{l}\text { S-03 } \\
8\end{array}$ & $\begin{array}{l}\text { b } \\
1\end{array}$ & 0 & 42 \\
\hline
\end{tabular}

la forme masses, suremployée par la CGT, passe de la fréquence 26 à la fréquence 8 entre les années 70 et les années 80, malgré l'inflation des textes caractéristique des derniers congrès cégétistes. Indice d'un changement en profondeur du discours, la chute générale de ce type de vocabulaire accompagne la chute constatée par ailleurs de travailleurs, action et lutte, ainsi que l'illustre le tableau 2.

Tableau 2:

Évolution des emplois de travailleurs, action et lutte

\begin{tabular}{|c|c|c|c|c|c|c|c|c|c|}
\hline & $\begin{array}{l}\text { CFDT } \\
73-79 \\
43042\end{array}$ & $\begin{array}{l}\text { CFDT } \\
82-88 \\
29704\end{array}$ & $\begin{array}{l}\text { CFTC } \\
71-79 \\
42266\end{array}$ & $\begin{array}{l}\text { CFTC } \\
81-90 \\
92239\end{array}$ & $\begin{array}{l}\text { CGT } \\
72-78 \\
127961\end{array}$ & $\begin{array}{l}\text { CGT } \\
82-89 \\
316352\end{array}$ & $\begin{array}{l}\text { FO } \\
71-80 \\
69613\end{array}$ & $\begin{array}{l}\text { FO } \\
84-89 \\
29942\end{array}$ & $\begin{array}{l}F \\
71-90 \\
751119\end{array}$ \\
\hline $\begin{array}{l}\text { travail } \\
\text {-leurs }\end{array}$ & $\begin{array}{l}\mathbf{S}+\mathbf{3 3} \\
351\end{array}$ & $\begin{array}{l}\text { b } \\
109\end{array}$ & $\begin{array}{l}\text { S+04 } \\
217\end{array}$ & $\begin{array}{l}\text { S-62 } \\
115\end{array}$ & $\begin{array}{l}\mathbf{S}+\mathbf{5 3} \\
866\end{array}$ & $\begin{array}{l}\text { S-05 } \\
1184\end{array}$ & $\begin{array}{l}\text { S-08 } \\
204\end{array}$ & $\begin{array}{l}\text { S-19 } \\
40\end{array}$ & 3086 \\
\hline action & $\begin{array}{l}S+80 \\
351 \\
\end{array}$ & $\begin{array}{l}\mathbf{S}+\mathbf{3 7} \\
204 \\
\end{array}$ & $\begin{array}{l}\text { S-03 } \\
81 \\
\end{array}$ & $\begin{array}{l}\text { S-11 } \\
145\end{array}$ & $\begin{array}{l}\text { S-03 } \\
281 \\
\end{array}$ & $\begin{array}{l}\text { S-15 } \\
627\end{array}$ & $\begin{array}{l}\mathbf{S}-\mathbf{0 3} \\
136\end{array}$ & $\begin{array}{l}\text { S-03 } \\
53\end{array}$ & 1878 \\
\hline lutte & $\begin{array}{l}\mathbf{S + 1 9} \\
117\end{array}$ & $\begin{array}{l}\text { S-02 } \\
20\end{array}$ & $\begin{array}{l}\text { S-12 } \\
8\end{array}$ & $\begin{array}{l}\text { S-21 } \\
25\end{array}$ & $\begin{array}{l}\text { S+07 } \\
195\end{array}$ & $\begin{array}{l}S+07 \\
416\end{array}$ & $\begin{array}{l}\text { S-11 } \\
27\end{array}$ & $\begin{array}{l}\text { S-06 } \\
10\end{array}$ & 818 \\
\hline
\end{tabular}

Ce second tableau présente un double intérêt. D'une part il confirme la tendance cégétiste et cédétiste à un emploi plus important en première période de classe, masse, travailleurs, action et lutte; d'autre part, à l'exception d'action et de lutte, formes restées très positives au début des années 80 respectivement à la CFDT et à la CGT et d'une évolution indécise à $\mathrm{FO}$, il confirme l'effondrement en seconde période de tout un pan du vocabulaire syndical de haute fréquencel1. En effet de nombreux termes accompagnent leur chute, tandis que d'autres les remplacent dans la hiérarchie des emplois: travailleurs se trouve supplanté par salariés et chômeurs, lutte(s) par négociation(s), transformation par évolution ou mutation, socialisme par modernisation, capitalisme par entreprises, autogestion par garanties, insertion, etc. Un discours révolutionnaire (propre davantage au 
clan CGT-CFDT) s'opposait-il au départ à un discours contractuel (clan FO-CFTC)? Estce ce dernier qui, dans les années récentes, l'emporterait totalement, signe d'une évolution générale qui irait de l'offensive et du projet de société à la défensive et au réalisme économique ? Contentons-nous de constater: on peut suivre cette mutation de congrès à congrès et confédération par confédération. La fracture s'échelonne, pour les mots comme pour les centrales, de 1981 à 1985. Masse et classe seraient donc des 'marqueurs' ou des 'indices de rappel' d'une même idéologie révolutionnaire en voie de déperdition lexicale ? Ce serait aller trop vite en besogne. Car les deux termes ne jouent pas de la même manière en contexte.

\section{ÉTUDE DU RÉSEAU : L'OPPOSITION CLASSE / MASSE}

\section{1 - Classe, masse comme déterminés}

À l'exception du segment déterminatif «de classe», «la classe» est obligatoirement déterminée par une suite, souvent très stéréotypée (une centaine de ses occurrences, sur les 496 où elle possède une pertinence sociologique, en sont affectées); il en est de même pour la forme plurielle et pour masse( $s$ ). Examinons les principales de ces déterminations (à $F>=2$ ).

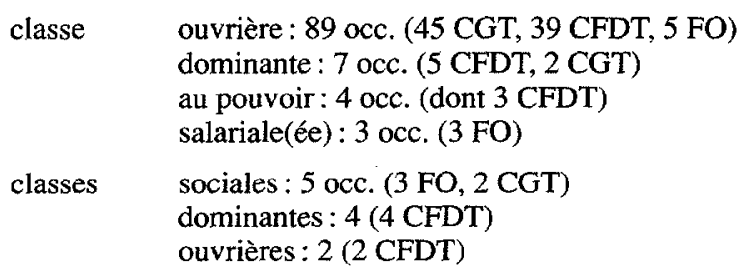

À l'unique exception de la lexie classe ouvrière (89 occurrences à elle seule), classe est un terme relativement peu déterminé. On dirait qu'une stéréotypie uniformisante a passé sur des dénominations qui furent si variées au XIX ${ }^{\mathrm{e}}$ siècle et jusqu'en 1936. Les locutions classe des travailleurs, des prolétaires, des ouvriers, laborieuse, prolétarienne, populaire, pauvre, indigente, ou moyenne, bourgeoise, capitaliste, patronale, dirigeante, régnante, aisée, etc. et leur pluriel semblent avoir disparu des congrès syndicaux. Le syntagme «classe des...», par exemple, n'obtient aucune occurrence dans les résolutions confédérales. Une telle simplification permet une dichotomie absolue. La société semble se résumer, dans les résolutions de congrès cégétistes et cécédistes de la première période tout au moins, à l'affrontement d'une classe ouvrière et d'une classe dominante ou au pouvoir.

Masse n'entraîne pas la même schématisation sociale. Ses déterminatifs sont d'abord plus variés, nuançant l'emploi d'un terme moins figé et plus disponible quant à sa contextualisation. A F>=2, on trouve en effet :

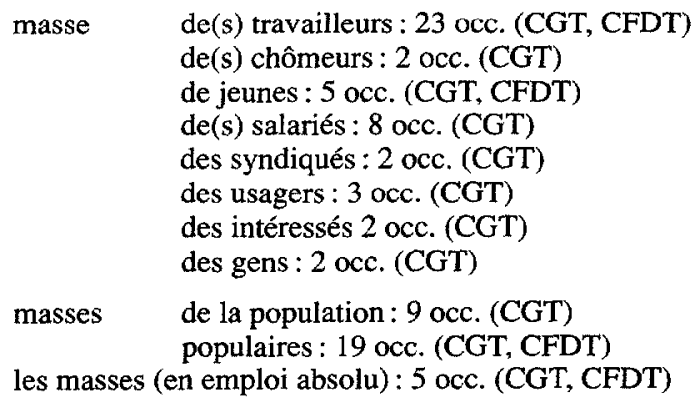

les masses (en emploi absolu) : 5 occ. (CGT, CFDT) 
En outre, chacune de ces lexies est susceptible de variations, par l'apport d'intrus adjectivaux: «la grande masse des travailleurs», «une masse croissante de travailleurs», «les grandes masses de la population», «cette masse grandissante de sans-emploi», «la masse des petits salariés», «une masse énorme de jeunes», «les larges masses populaires», etc.

Cette double différence de détermination entre classe et masse nuance davantage encore leur fonctionnement lorsqu'on interroge les termes en tant que déterminants. Il s'agit le plus souvent des syntagmes adjectivaux «de classe» et «de masse».

\section{2-Classe, masse comme déterminants}

Ou bien le terme fait référence aux salariés que le syndicalisme représente ou bien il désigne l'adversaire: classe de soi/classe de l'autre. À une exception près («antagonismes de classe»), il n'y a pas de terme bivalent, à la CGT comme à la CFDT, sauf bien sûr au pluriel. Les groupes nominaux peuvent se répartir en deux ensembles dont le déséquilibre en fréquence vient de ce que le discours syndical est toujours davantage axé sur soi que sur l'autre. Nous excluerons de ce tableau le segment «de classe et de masse» et son inverse, traités à part.

\section{la classe de soi}

acte collectif de classe (CFDT)

action de classe (CGT)

affrontement de classe (CGT)

bases(s) de classe (CGT)

bataille de classe (CGT)

caractère de classe (CGT)

conception de classe (CGT)

combat de classe (CGT)

conscience de classe (CFDT, CGT)

contenu de classe (CGT)

dimension de classe (CFDT)

orientation(s) de classe (CGT)

unité de classe (CGT)

lutte(s) de classe (CGT, CFDT)

combat idéologique de classe (CFDT)

intérêts de classe (travailleurs) (CGT)

identité de classe (CFDT))

enjeu( $x$ ) de classe (CGT)

organisation (syndicale) de classe (CGT)

pratique... de classe (CGT)

principes de classe (CGT)

positions de classe (CGT)

rassemblement de classe (CFDT)

stratégie de classe (CFDT)

syndicalisme de classe (CGT)

syndicat de classe (CGT)

solidarité (internationale) de classe (CGT)

terrain de classe (CGT, FO)

analyse de classe (CFDT)

action syndicale de classe (CGT)

action syndicale internationale de classe (CFDT)

point de vue de classe (CGT)

idées de classe (CGT)

\section{la classe de l'autre}

adversaire de classe (CGT, CFDT)

au service de la classe dominante (CFDT)

caractère de classe de la justice (CFDT)

caractère de classe de la fiscalité (CGT)

caractère de classe des positions dites libérales

(CFDT)

esprit de classe et de caste (FO)

intérêts de classe des monopoles (CGT)

pouvoir de la classe dominante (CFDT)

prédominance de la classe au pouvoir (CFDT)

offensive de la classe dominante (CFDT)

fiscalité de classe (FO)

nature de classe (CGT)

collaboration de classe (CGT)

choix de classe (CGT)

intérêts de classe et de caste (CGT)

politique de classe (CGT)

privilèges de classe (CGT)

raisons de classe (CGT)

Il est visible que nous sommes ici à l'intérieur du lexique de la CGT. La CFDT et, à un très moindre degré, FO s'y font une place, en particulier lorsqu'il s'agit de la «classe de l'autre». La CFTC, elle, se veut absente du terrain : la lutte de classe ne la concerne pas. 
Deux éléments initiaux seulement sont communs aux deux ensembles: intérêts et caractère. Les autres termes sont réservés à l'usage d'un seul camp, lutte(s) de classe en particulier, n'apparaît qu'en relation avec la classe ouvrière, malgré sa très haute fréquence (78 occ.), lutte étant un terme de travailleurs, jamais de patronat.

Les formes déterminées à la CGT et à la CFDT par le de classe propre à la classe ouvrière dessinent trois champs d'expression: celui de la dynamique populaire dans le conflit social, celui des rapports internes à la classe ou au syndicalisme, celui des principes de cette action et de cette organisation.

- Dynamique conflictuelle : lutte(s), action, acte, affrontement, bataille, combat, défense des intérêts, enjeu( $x$ )... de classe;

- Organisation et analyse: pratique, rassemblement, syndicalisme, unité, organisation(s), identité, stratégie, solidarité, analyse... de classe;

- Principes «de classe»: positions, base(s), orientation(s), dimension, caractère, principes conception, terrain, contenu, conscience, prise de conscience, idées, points de vue... de classe.

Dans presque la moitié des cas, c'est à une expression nominale plus longue que nous avons affaire; l'enfilade des déterminations peut atteindre trois, quatre étapes, voire cinq étapes où le segment de classe occupe la dernière position. Evoquons quelques exemples des enchaînements les plus longs :

$\begin{array}{llll}\text { un moyen } & \text { de la pratique } & \text { de la lutte } & \text { de classe } \\ \text { analyse } & \text { des conditions actuelles } & \text { de la lutte } & \text { de classe } \\ \text { méthodes } & \text { d'incitation } & \text { à la collaboration } & \text { de classe } \\ \text { d'appui } & \text { à la poursuite } & \text { de l'action } & \text { de classe } \\ \text { du rapport } & \text { de forces } & \text { dans la lutte } & \text { de classe } \\ \text { place } & \text { des organisations } & \text { de collaboration } & \text { de classe } \\ \text { cet outil } & \text { d'action syndicale } & \text { de lutte } & \text { de classe } \\ \text { le jeu } & \text { de cette politique } & \text { de collaboration } & \text { de classe, etc. }\end{array}$

Ce type de discours à déterminations en rebondissements caractérise aussi le segment de masse. Celui-ci joue autant dans le discours de la CGT que dans celui de la CFDT (avec quelques cas à FO), avec des expressions comme «action(s) de masse», «lutte(s) de masse», «organisation de masse», «sport de masse», «éducation de masse», «caractère de masse» et, bien sûr, «syndicalisme de masse». Mais certains groupes déterminants formés sur masses caractérisent la seule CGT:

$\begin{array}{llll}\text { besoins } & \text { des travailleurs } & \text { et des grandes masses } & \text { de la population } \\ \text { conditions de vie } & \text { des travailleurs } & \text { et des grandes masses } & \text { de la population } \\ \text { à celui } & \text { des travailleurs } & \text { et des grandes masses } & \text { de la population } \\ \text { aux besoins } & \text { des travailleurs } & \text { et des grandes masses } & \text { de la population } \\ \text { les luttes } & \text { des salariés } & \text { et des grandes masses } & \text { de la population } \\ \text { participation } & \text { des travailleurs } & \text { et des masses } & \text { populaires } \\ \text { intérêts } & \text { des travailleurs } & \text { et des masses } & \text { populaires } \\ \text { les besoins } & \text { des salariés } & \text { et des masses } & \text { populaires }\end{array}$

On dirait qu'au fur et à mesure que les séquences s'allongent la stéréotypie se renforce pour aboutir à un segment final archi-figé.

Celui-ci possède pourtant deux variantes: «de classe et de masse» et «de masse et de classe». Loin d'être un effet de hasard, celles-ci introduisent un clivage à l'intérieur du clan CGT-CFDT et jusqu'au sein de la CGT elle-même.

\section{ÉTUDE D'UNE SITUATION : UN CLIVAGE INTRACONFÉDÉRAL}

Malgré leur fréquence faible, les segments inversables qui utilisent dans une même séquence nominale les deux formes classe et masse selon deux combinaisons, ont une 
distribution statistiquement très significative : classe suivi par masse forme un couple caractéristique de la CGT (avec 16 occ. sur 25, en particulier dans la seconde période), alors que masse suivi par classe appartient au discours cédétiste (avec $20 \mathrm{occ}$. sur 26 , surtout durant la première période). Quant à FO et à la CFTC, elles ignorent totalement ces deux types d'expression. Le jeu qu'ils mènent ne les intéresse pas.

\begin{tabular}{|c|c|c|c|c|c|c|c|c|c|}
\hline & $\begin{array}{l}\text { CFDT } \\
73-79 \\
43042\end{array}$ & $\begin{array}{l}\text { CFDT } \\
82-88 \\
29704\end{array}$ & $\begin{array}{l}\text { CFTC } \\
71-79 \\
42266\end{array}$ & $\begin{array}{l}\text { CFTC } \\
81-90 \\
92239\end{array}$ & $\begin{array}{l}\text { CGT } \\
72-78 \\
127961\end{array}$ & $\begin{array}{l}\text { CGT } \\
82-89 \\
316352\end{array}$ & $\begin{array}{l}\text { FO } \\
71-80 \\
69613\end{array}$ & $\begin{array}{l}\text { FO } \\
84-89 \\
29942\end{array}$ & $\begin{array}{l}\text { Total } \\
71-90 \\
751119\end{array}$ \\
\hline $\begin{array}{l}\text { de classe } \\
\text { et de } \\
\text { masse }\end{array}$ & $\begin{array}{l}\text { b } \\
9\end{array}$ & 0 & 0 & 0 & $\begin{array}{l}\text { b } \\
3\end{array}$ & $\begin{array}{l}\mathbf{S}+\mathbf{0 2} \\
13^{* * *}\end{array}$ & 0 & 0 & 25 \\
\hline $\begin{array}{l}\text { de masse } \\
\text { et de } \\
\text { classe }\end{array}$ & $\begin{array}{l}\text { S+17 } \\
18^{*}\end{array}$ & $\begin{array}{l}\text { b } \\
2\end{array}$ & 0 & $\begin{array}{l}\text { S-02 } \\
0\end{array}$ & $\begin{array}{l}\text { b } \\
1\end{array}$ & $\begin{array}{l}\text { S-02 } \\
5^{* * * *}\end{array}$ & 0 & 0 & 26 \\
\hline
\end{tabular}

* On a inclus dans ces occurrences une variante cédétiste : «syndicalisme de masse, de classe».

** On a inclus trois variantes cégétistes : «de classe, de masse», «de classe, de masse et démocratique» et «de classe, de masse, démocratique et indépendant».

*** On a inclus deux variantes cégétistes «caractère de masse et caractère de classe» et «activité syndicale de masse sur des bases de classe».

Quelque chose se joue dans l'opposition statistique de ces deux enchaînements. Pour y voir plus clair, examinons un congrès de la CGT où ils se sont affrontés, sans en avoir l'air mais sans en perdre la chanson, au cours de la discussion qui a suivi le rapport du secrétaire général. Il s'agit de Montreuil, au 26 novembre 1985, congrès de bétonnage s'il en est un, puisque c'est à sa suite que les derniers socialisants du bureau national ont été éliminés. Suivons pas à pas les interventions orales ${ }^{12}$. Le président (Amiable) ouvre la séance en annonçant la couleur avec «syndicalisme de classe» et passe la parole à $\mathrm{H}$. Krasucki. Une première salve tourne notablement autour de masse (avec action syndicale de masse, la masse des gens, en masse, intervention de masse, action de masse et les grandes actions les plus massives). Une seconde s'annonce sous le signe de classe. Passent dans le discours lutte des classes et lutte de classe ( 2 fois), mais les mots se mêlent. On entend : résistance de masse et plus loin la masse des salariés, dans leur masse, plus loin encore action massive, la grande masse des salariés, travail syndical de masse, en même temps que la désignation de la 'classe des autres': classe dominante ( 2 fois), puis justice de classe, classe dirigeante (en contrepoint, la lexie classe ouvrière arrive deux fois, dont une en discours rapporté), que suivent terrain de classe, conscience de classe, lutte de classe, syndicalisme de classe... Bref, la série énoncée avec masse compte au total 12 occurrences; celle énoncée avec classe 13. Un tel mélange, si bien équilibré, serait-il le seul fait du hasard?

Une phrase d'H. Krasucki donne la clé des sens: «En France, c'est avant tout la CGT. Parce qu'elle est le syndicat qui se bat. Le syndicat de lutte et de rassemblement. Le syndicat de classe qui ne s'en laisse pas conter sur les réalités de la société capitaliste et le syndicat de masse qui s'adresse à tous les travailleurs salariés.» On remarque, dans cette phrase, le parallélisme entre «lutte», «société capitaliste» et «de classe» d'une part et «rassemblement», «tous les travailleurs salariés» et «de masse» d'autre part. Retenons aussi la hiérarchie des thèmes: classe précède masse. 
Plus intéressant encore, le débat qui va suivre. Les délégués s'expriment les uns après les autres. Celui du syndicat Renault de Douai parle «des étapes à franchir d'abord par l'avant-garde et très vite par la grande masse» (grâce à un autre mot, avant-garde, l'ordre des deux thèmes semble respecté, mais c'est masse qui est prononcé...). Avec la Fédération des transports, $c$ 'est «syndicalisme de classe» qui arrive, seul. En revanche, les imprimeurs de La Chapelle d'Herblay ne jouent pas le jeu consensuel et prônent «un syndicalisme de masse et de classe». Réplique de l'Assistance publique de Paris : «Ce n'est pas perdre sa conscience de classe que de dénoncer les responsables [de la «casse» du secteur].» Les PTT (A. Le Guern) glissent dans leur discours à la fois «activité catégorielle de masse» et l'affirmation que «la CGT est restée fidèle à sa classe». Net coup de barre avec M. Coquis (Plastiques de Giens), toujours contre la «casse» des gouvernants : «Pour casser les usines, il faut casser ce qui existe, il faut casser leur syndicat de classe, la CGT.» F. Dumez (UD du Nord) relance la volonté d'équilibre manifestée par H. Krasucki - et dans le bon ordre - : «Nous avons toujours refusé d'opposer le principe de classe et le principe de masse.» Ordre inverse chez Véronèse (délégation à l'A.N.) : on entend l'expression «un syndicalisme authentiquement indépendant, de masse, de classe». $\mathrm{Ce}$ qui n'est que discours critique en creux ( $c f$. l'allusion «authentiquement indépendant»...) devient une option claire chez G. Gaumé (secrétaire socialisant, qui sera démissionnaire), lequel, au nom d'un «syndicalisme démocratique de masse et de classe», demande de «renforcer et maintenir le caractère de masse de la CGT» pour «une CGT de masse», ajoutant «ces dernières années, la CGT a connu un certain rétrécissement».

À l'exception de ces interventions, l'enjeu n'est jamais dit: La bonne formule avait été lancée dès le début par le secrétaire général : «syndicalisme de classe et de masse»; c'est elle qui fera le fond de la quasi-totalité des discours et des motions présentées. La petite minorité favorable à la combinaison inversée se fera éliminer sans qu'on y prenne même garde, les discours parlant d'autre chose, axés qu'ils sont à cette époque sur la «casse» gouvernementale et patronale. Derrière l'ordre de deux mots, le pouvoir...

L'examen des prises de parole au congrès cégétiste de Montreuil (1985) montre ainsi que des stéréotypes de surface révèlent des options de fond en conflit: il y a ceux qui disent «de classe» (communistes sectaires); ceux qui disent «de masse» (socialisants); ceux enfin, les plus nombreux, qui s'efforcent à l'équilibre, aux variantes, mais en respectant un ordre de priorité dans la mise en séquence des deux options, suivant l'exemple d'H. Krasucki.

Qu'est-ce donc que le sens d'un mot? Comment dire le pouvoir derrière le mariage de deux stratégies, voire idéologies, syndicales différentes? Classe spécifie le caractère socio-économique de la lutte; masse appelle à la réunion de tous les «travailleurs» sans distinction. Classe est sans doute encore senti comme signe marxiste et surtout partisan; masse peut-être encore comme héritage anarcho-syndicaliste ou socialisterévolutionnaire.

Le sens de ces mots est dans leur fonction. À la différence de classe qui spécifie et, aujourdhui, restreint, le terme de masse sert à élargir les perspectives du regroupement; il est senti d'une orbite sémantique plus englobante que celle de travailleurs salariés, donc plus généralisante que celle de classe. Voilà pourquoi l'ajout du segment «de masse» à «de classe» n'est pas qu'une figure rhétorique de la redondance, et la place préférentielle de masse en première position à la CFDT ou en dernière à la CGT (et surtout dans le discours orthodoxe de la CGT) est loin d'être sans pertinence. On pourrait même dire que la position d'antériorité en séquence dévolue à classe signifie, pour les cadres communistes de la CGT, que la généralisation de l'action ne doit se faire qu'à partir d'un noyau central homogène. À la limite, le segment de classe ne s'identifierait-il pas pour eux au «Parti»? (Le mot d'avant-garde a été prononcé à sa place...) Évoquant «l'indépendance 
de la CGT», H. Krasucki la décrit comme «placée sur des positions de classe, tout en ayant un contenu de masse très large». Synthèse, mais orientée.

Masse serait donc au service d'une stratégie de rassemblement populaire, alors que classe resterait le signe intransigeant d'une idéologie 'classiste' ou d'une direction partisane. À chacun son rôle dans la fermeture/ouverture du syndicalisme.

\section{Notes}

1. Marie-France Piguet, Le vocabulaire de la division sociale et la formation du concept de «classe" (I7581928), thèse doctorale, soutenue le 25 septembre 1993 à l'Université Paris-3. Nous apprenons en particulier que l'usage structurel de classe est entièrement dû aux Physiocrates. En effet, à l'inverse de Quesnay, A. Smith exprime son concept socio-économique à l'aide des anciens termes hiérarchistes d'order et de rank. Lorsque class apparaît dans sa Richesse des nations, c'est à travers son commentaire des Physiocrates. Renversement des perspectives : class, chez Smith, serait un calque de l'usage français. La comparaison des traductions le prouve. Marx n'a eu qu'à lire la traduction 'classiste' de Garnier...

2. Cf. «En son nom [G. Sand] on provoquait aux luttes de classes», écrit Louis Reybaud (Jérôme Paturot à la recherche de la meilleure des républiques, Paris, Michel Lévy, 1848).

3. Constat fait sur $L ' E ́ c h o$ de la Fabrique, journal «industriel et littéraire» des canuts, Lyon, impr. Jérôme Perret, 1831-1834.

4. Cf. François Perroux, Masse et classe, Paris, Casterman, 1972.

5. «C'est par la grève que la masse ouvrière entre dans la lutte de classe» (Pierre Monatte, Congrès «syndicaliste-révolutionnaire» d'Amsterdam, 1907).

6. Rosa Luxembourg, L'expérience belge. Réponses à Vandervelde, 1913.

7. UMR «Lexicométrie et textes politiques», unité constitutive de l'INaLF, CNRS, et rattachée à l'École normale supérieure de Fontenay-Saint-Cloud (directeurs : Annie Geffroy, Pierre Lafon). Une équipe du laboratoire a publié en 1982 La parole syndicale. Étude du vocabulaire confédéral des centrales ouvrières françaises (1971-1976), Paris, PUF, (Coll. «Politique d'aujourd'hui»; elle rédige actuellement un Dictionnaire des fréquences syndicales, à partir du corpus décrit ici. Autres publications : «Discours syndical ouvrier en France», Mots. 14, mars 1987 et «Un demi-siècle de vocabulaire syndical», Mots, 36, septembre 1993.

8. Méthode qui consiste dans la probabilisation de toutes les fréquences locales obtenues pour les formes et les segments d'un corpus selon la partition étudiée. Cf. Pierre Lafon, Dépouillements et statistiques en lexicométrie, Genève-Paris, Slatkine-Champion, 1984, pp. 45-85, et André Salem, Pratique des segments répétěs, Paris, Klincksieck, 1987, pp. 207-232.

9. Les indices du tableau sont à lire ainsi : S+ (spécificité positive ou suremploi significatif à un seuil de $5 \%$ ), b (distribution banale, dont la probabilité atteint ou dépasse le seuil de $5 \%$ ), S- (spécificité négative ou sous-emploi), absence de signe (sans significativité). Le chiffre qui suit l'indice représente la valeur absolue de l'exposant négatif de la probabilité : ainsi, S+ suivi de 02 s'interprète comme spécificité positive de l'ordre du centième mais inférieure à $5 \%$. Le chiffre indiqué en dessous de l'indice est celui de la fréquence locale.

10. Quelques précautions sont en effet nécessaires quant aux comptages, car certaines fréquences comportent des intrus dont il convient de prendre la mesure. C'est ainsi qu'on trouvera classe en emploi scolaire dans l'unique occurrence de la CFTC ( 3 fois à la CGT et 1 à FO). Le pluriel classes est scolaire 1 et 2 fois à la CFTC, 4 et 7 fois à la CGT, 8 et 3 fois à FO. Pour masse, on dépiste 10 occurrences au total du type «masse des salaires / des impôts». Quant à masses, ses deux occurrences à la CFTC concernent les «masses financières». L'incidence de ces décomptes est très faible sur le calcul des probabilités, sauf pour FO et la CFTC, ce dont il faut tenir compte dans les commentaires.

11. Sur cette mutation générale, $c f$. Mots 36, articles d'André Salem : «De travailleurs à salariés. Repères pour une étude de l'évolution du vocabulaire syndical (1971-1990)», pp. 74-83, Josette Lefêvre: «CFDT 19731992. L'éclatement d'un champ lexical», pp. 84-100, M. Tournier : «Travailleur aux prises avec l'histoire», pp. 119-124.

12. Notes prises en séance sur les interventions orales. 